\title{
Representações sociais das infecções hospitalares elaboradas pelos profissionais de saúde
}

\author{
Social representations of hospital infections elaborated by health professionals
}

Representaciones sociales de las infeciones hospitalares elaboradas por profesionales de salud

\section{Maria Eliete Batista Moura', Fabrício Ibiapina Tapety', Carmen Milena Rodrigues S. Carvalho', José Nazareno Pierce de Oliveira', Francisca Tereza Coelho Matos', Luana Kelle Batista Moura'}

'Faculdade NOVAFAPI. Teresina, PI

Submissão: 19/03/2008

Aprovação: 3 1/07/2008

\section{RESUMO}

O estudo objetivou apreender e analisar as Representações Sociais das Infecções Hospitalares elaboradas pelos profissionais e estudantes de saúde de um hospital público de Teresina-PI. Pesquisa descritiva, com abordagem Qualitativa. Os dados foram produzidos a partir do teste de associação livre de palavras, e processados pelo software Tri-Deux Mots, Que deram origem a 563 palavras como respostas para os estímulos: Infecção hospitalar, procedimentos invasivos, esterilização de material, precaução padrão. O posicionamento dos sujeitos da pesquisa frente à infecção hospitalar foi evidenciado com evocações, tais como: paramentação, sondagem e mãos. $\mathrm{O}$ resultado reforça a ênfase dada pelos profissionais da saúde à paramentação para sua própria proteção e a do paciente durante procedimentos invasivos, ressaltando a importância do cuidado com as mãos.

Descritores: Infecção hospitalar; Psicologia social; Prevenção.

\section{ABSTRACT}

This study has the aim to analyze the Social Representation of the Hospital Infections elaborated by health professionals and health students of a public hospital of Teresina-PI. The study has a Quantitative and descriptive approach. The data was produced based on the free association of words test and processed by the Tri-Deux Mots software gave origin to 563 words as response to the following stimulus: Hospital infection, invasive procedures, sterilization of materials and standard. The positions of the subjects regarding hospital infection was demonstrated with words such as: paramentation, probing and hands. The results reinforce the emphasis given by the health professionals to the paramentation for his own and the patient protection during invasive procedures, pointing out the importance to the hands hygiene.

Descriptors: Hospital infection; Social psychology, Prevention.

\section{RESUMEN}

El estudio objetivó aprehender e analizar las Representaciones Sociales de las Infecciones Hospitalarias elaboradas por los profesionales e estudiantes de salud de un hospital estatal de Teresina-PI. Peseuisa descriptiva, con abordaje cualitativa. Los dados fueron producidos a partir de teste de asociación libre de palabras, y procesados por el software Tri-Deux Mots, Que dieron origen a 563 palabras como respuestas para los estímulos: Infección hospitalario, procedimientos invasivos, esterilización de material, precaución padrón. El posicionamiento de los sujetos de la investigación frente a la infección hospitalario fue evidenciado con evocaciones, tales como: paramentación, sondaje y manos. El resultado refuerza la énfasis dada por los profesionales de la salud a la paramentación para su propia protección y la del paciente durante procedimientos invasivos, resaltando la importancia del cuidado con las manos.

Descriptores: Infección hospitalaria; Psicología social; Prevención. 


\section{INTRODUÇÃO}

Construindo o objeto de estudo das Representações Sociais

As infecções hospitalares (IH) existem desde Que surgiram os hospitais (século XIX), devido à elevada incidência de doenças epidêmicas, Que acometiam às comunidades pobres e, também, às precárias condições de higiene e de saneamento em Que vivia a população ${ }^{(1)}$.

No Brasil, o Ministério da Saúde (MS), na Portaria no 2.616 de 12/05/1998, define IH como a infecção adQuirida após a admissão do paciente na unidade hospitalar e eue se manifesta durante a internação ou após a alta, Quando puder ser relacionada com a internação ou procedimentos hospitalares ${ }^{(2)}$.

Nestas últimas décadas, muitos temas na área da saúde tiveram grande destaque, com ênfase nas áreas de atuação dos profissionais da saúde, tornando-se de domínio público. Uns porQue dizem respeito ao direito da cidadania e outros poreue se relacionam com os problemas da prática e da ética profissional, Que devem ser cuidadosamente revisados, tratados e contornados a fim de zelar por benefícios sociais ou de cunho político social.

Dessa forma, este estudo tem como objeto as Representações Sociais da Infecção Hospitalar elaborada pelos profissionais e estudantes da área da saúde, para conhecer como representam a problemática das $\mathrm{IH}$ e as políticas de prevenção e controle adotadas.

A Teoria das Representações Sociais (TRS) é uma teoria sobre a construção social. A forma como as pessoas pensam o seu universo, resulta em um conhecimento socialmente construído por meio de discursos nos grupos ${ }^{(3)}$.

As RS parecem ser particularmente úteis para a análise, a compreensão e a intervenção sobre os grandes problemas sociais atuais: a saúde, a doença e, em particular, a disseminação da AIDS, Que poderá ocorrer pelas constantes infecções cruzadas nos ambientes dos serviços de saúde( ${ }^{(4)}$.

O objeto das RS está inscrito num contexto ativo e dinâmico Que é concebido pela pessoa ou coletividade como extensão de sua conduta. É, nesse sentido, uma preparação para a ação(5).

As RS se constituem em um saber prático, Que é lembrado sempre Que se necessita realizar uma ação, como, por exemplo, a observância de um plano terapêutico ou uma mudança de conduta com relação às práticas sociais. As RS regem nossas relações com o mundo e com os outros e organizam nossas comunicações e condutas. Assim, o seu estudo pode contribuir para Que se faça uma análise Que contemple mais amplamente a complexidade das práticas sociais ${ }^{(6,7)}$.

Dessa forma, ainda na atualidade, o Programa de Prevenção e Controle das IH do Governo Federal, continua tomando como base as tradições positivistas e assim apresenta-se com capacidade limitada para modificar os indicadores de IH nos serviços de saúde do país e não contribui para mudanças de atitudes dos profissionais de saúde que realizam os procedimentos na assistência aos clientes.

A maioria dos programas do Governo Federal não leva a mudança de atitudes, pois supõe Que os indivíduos que participam desses programas sejam passivos e não detenham saberes e valores prévios, Que conduzam suas ações. Há a necessidade da adoção de novos referenciais e de novas metodologias eue superem as tradições positivistas, pois essas fragmentam a realidade em variáveis, desenhando dimensões restritas e isoladas das relações psicossociais e culturais mais amplas ${ }^{(8)}$.

Dessa forma, com base nessa problemática, definiram-se como objetivos: apreender e analisar as Representações Sociais das Infecções Hospitalares elaboradas pelos profissionais e estudantes de saúde.

\section{METODOLOGIA}

Trata-se de uma pesQuisa descritiva, com abordagem eualitativa, por ser mais adeQuada para investigar a problemática apresentada, para estudar os fenômenos sócio-culturais das representações sociais relacionadas com a infecção hospitalar, fundamentada na Teoria das Representações Sociais.

O cenário da pesquisa foi um hospital público, de grande porte, localizado em Teresina. Os sujeitos foram 68 profissionais e estudantes da área da saúde. Os dados foram produzidos a partir do teste de associação livre de palavras, e processados pelo software Tri-Deux Mots originando um conjunto de 563 palavras como respostas para os estímulos: infecção hospitalar, procedimentos invasivos, esterilização de material, precaução padrão. Dessas, 166 com significados diferentes.

O teste de associação livre de palavras, originalmente desenvolvido por Jung na prática clínica e adaptado no campo da psicologia social, vem sendo amplamente utilizado nas pesQuisas sobre Representações Sociais. O teste de associação livre de palavras trata-se de uma técnica projetiva orientada pela hipótese de Que a estrutura psicológica do sujeito torna-se palpável através das manifestações de condutas de reações, evocações, escolhas e criações, constituindo-se em índices reveladores do conjunto da personalidade ${ }^{(9)}$.

O instrumento se estrutura sobre a evocação das respostas dadas a partir de estímulos indutores. Tal estímulo consistiu em uma palavra previamente elaborada de acordo com a relação estabelecida entre os sujeitos da investigação e o objeto a ser pesquisado ou objeto representado. Esse termo indutor foi apresentado aos sujeitos, solicitando-lhes Que associassem prontamente uma ou mais palavras (ou expressões) Que lhes viesse à mente, esclarecendo Que Quanto menor o tempo gasto pelos sujeitos para elaboração das respostas, maior a validação dos resultados da pesQuisa. Foi também desestimulada a utilização de frases longas ou conceitos intelectualmente mais elaborados.

$\mathrm{Na}$ codificação dos dados, a primeira etapa consistiu na elaboração do dicionário correspondente ao estímulo. Foram agrupadas, separadamente, em areuivos, todas as respostas evocadas por toda a amostragem referente ao estímulo indutor. Em seguida, as respostas foram dispostas em ordem alfabética e posteriormente reagrupadas por similaridade semântica(9).

Posteriormente, organizou-se um banco de dados constituído pelas variáveis de palavras dispostas em linhas horizontais, codificadas e agregadas ao número referente aos estímulos indutores ( I - Infecção Hospitalar 2 - Procedimentos invasivos 3 - Esterilização de Material 4 - Precaução Padrão) e pelas variáveis fixas (sexo, idade, escolaridade) dispostas nas três primeiras colunas do banco de dados.

Uma vez organizados os dicionários e o banco de dados, os resultados do teste de associação livre de palavras foram processados no software Tri-Deux Mots; indicado para tratamento 
de Questões abertas e fechadas, e interpretados por meio da Análise Fatorial de Correspondência (AFC). Esse software foi desenvolvido para processamento estatístico de um volume de dados Que manualmente seria penoso e demorado. O mesmo permite verificar correlações entre grupos, assim como visualizar as relações de atração e afastamento entre os elementos do campo representacional a propósito de determinado objeto ${ }^{(9)}$.

A AFC avalia as correlações (positivas e negativas) existentes entre diferentes grupos de caracteres, colocando em evidência os campos semânticos definidos pelas palavras associadas aos estímulos e à sua contribuição para a construção dos fatores ou eixos Que constituem o plano fatorial. É ideal para explicar as representações sociais obtidas através do Teste de Associação Livre de Palavras, o Que enfoca as variáveis fixas ou sócio-demográficas (em coluna) e as variáveis ativas ou de opiniões (em linha), Que se confrontam e se revelam, graficamente, na representação do plano fatorial.

O gráfico Que foi construído a partir desse processo, corresponde às representações ou adjetivos Que os sujeitos evocaram a respeito do objeto em estudo, isto é, ao mapa dos agrupamentos e oposições entre os adjetivos associados à infecção hospitalar. Apresenta, portanto, resultados Que permitiram uma avaliação estatística dos dados no Que concerne à freQüência (importância de contribuição das modalidades na construção dos fatores), e a representação gráfica das variações semânticas na definição do campo espacial.

Para análise do material final deste processo foram consideradas as modalidades Que obtiveram a contribuição por fator superior a duas vezes a média, Que corresponde às modalidades ou palavras Que contribuíram com maior significação na construção dos fatores. A média é proveniente da divisão de 1000 pelo número de modalidades com significados diferentes, Que, no caso específico, corresponde a 166 . Em seguida, multiplica-se por 2 o resultado advindo desta divisão $(6,02)$, obtendo-se, assim, o valor superior a duas vezes a média $(12)^{(9)}$.

Desse modo, configuraram-se graficamente as evocações relativas aos estímulos indutores, revelando-se aproximações e oposições das modalidades analisadas através da Análise Fatorial de Correspondência, definindo assim, a objetivação da infecção hospitalar.

\section{RESULTADOS E DISCUSSÃO}

O fenômeno da Infecção Hospitalar pela ótica das

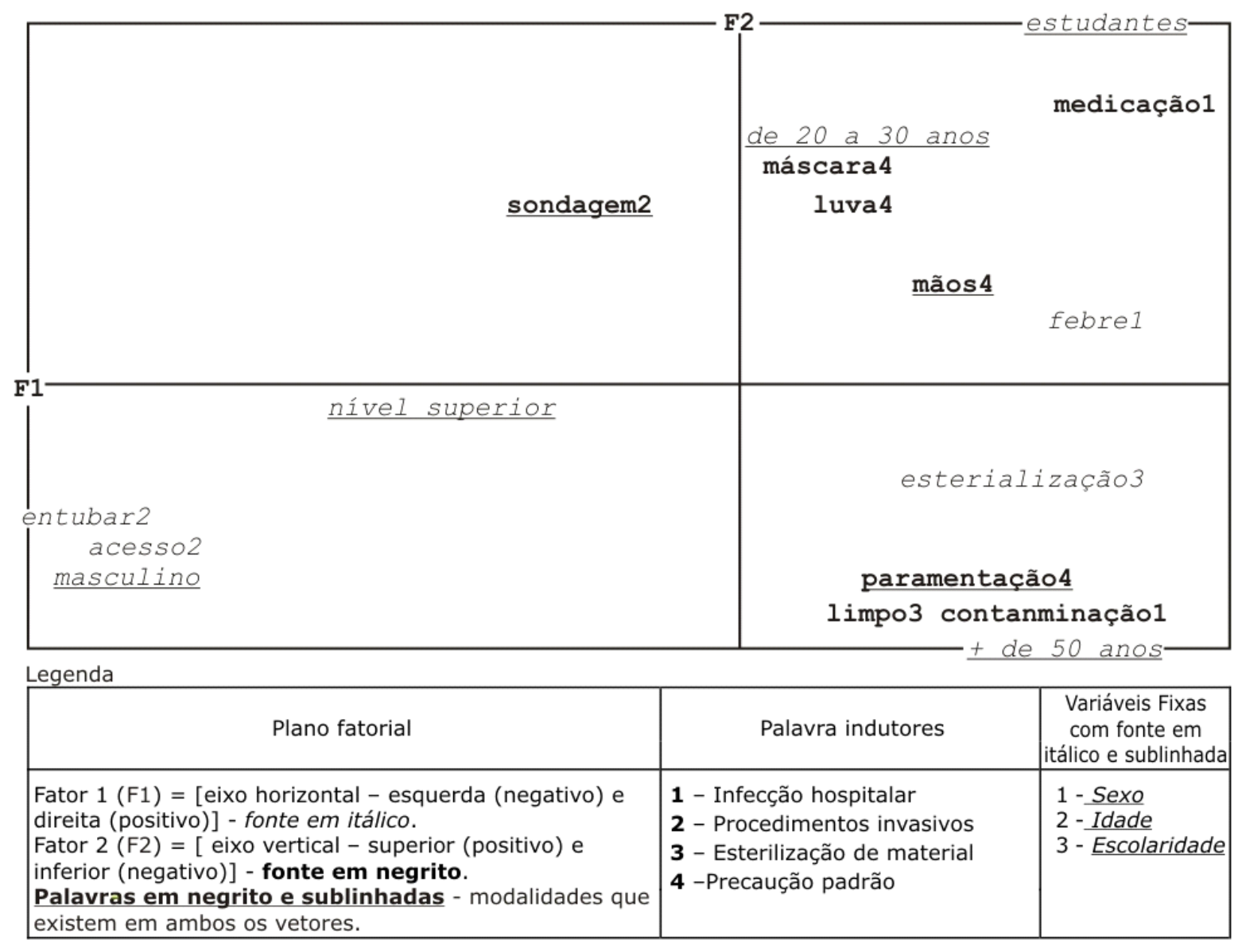

Percentual de contribuiçôes no $\mathrm{FI}=41,5 \% \% \mathrm{~F} 2=31,1 \%$

Total de contribuições dos dois fatores $=72,6 \%$

Total de Palavras $=563$

Total de Palavras diferentes $=166$

Gráfico 1. Representação gráfica do plano fatorial sobre infecções hospitalares. 
Representações Sociais possibilita edificação de conceitos, explicações e afirmações Que se originam no cotidiano através das comunicações interindividuais e do conhecimento socialmente elaborado e compartilhado. $\mathrm{O}$ conhecimento vincula-se às construções sociais influenciados pela cultura (opiniões, atitudes e estereótipos), pelos grupos sociais e pela história. Portanto, as Representações Sociais contribuem para a formação de condutas e orientação das comunicações; podem ser entendidas como uma teoria do "senso comum", designando uma forma de pensamento social $^{(10)}$

Assim, as Representações Sociais são uma "preparação para a ação"(10); elas são dinâmicas, em face de suas capacidades para ultrapassarem os limites do domínio psicossociológico, intervindo na definição da identidade individual, social e na construção de objetos; é a partir das RS Que o "indivíduo sofre a pressão das mesmas dominantes na sociedade, e assim, nesse meio, Que pensa e exprime seus sentimentos"(6).

No gráfico 1, observa-se o conteúdo das representações sobre Infecção Hospitalar, tornando-se possível, a partir da objetivação configurada no campo representacional, fazer a leitura e interpretação das modalidades de opinião pelas palavras evocadas, correlacionando-as com as variáveis fixas organizadas segundo os eixos ou fatores (FI e F2). Apresenta-se com fonte itálico as modalidades Que compõem o fator $\mathrm{FI}$, com fonte em negrito as modalidades do fator F2 e com fonte em negrito e sublinhado as modalidades comuns aos dois fatores FI e F2. E destaca-se com fonte itálico sublinhado a variável fixa.

\section{Fator I - Lado Esquerdo (negativo)}

Destacam-se, no gráfico do lado esQuerdo (negativo) do fator I (eixo horizontal com fonte em itálico) as representações sociais da infecção hospitalar e procedimentos invasivos, elaboradas por profissionais e estudantes da área da saúde do sexo masculino e profissional de nível superior.

Segundo esse grupo de sujeitos, infecção hospitalar é sinonímia de sondagem, acesso e entubar. Através dessas modalidades os profissionais e estudantes da área da saúde reconhecem que os procedimentos invasivos, por ultrapassarem as barreiras naturais do organismo, favorecem o surgimento e disseminação da infecção hospitalar ${ }^{(11,12)}$.

\section{Fator I- Lado Direito (positivo)}

No lado oposto (direito positivo) do mesmo fator (Fl), observase a delimitação do agrupamento das representações sociais sobre os estímulos infecção hospitalar, esterilização de materiais e precaução padrão, constituídas por profissionais e estudantes da área da saúde na faixa etária de 20 a 30 anos e com mais de 50 anos.

Neste lado do eixo, infecção hospitalar é sinonímia de febre, e precaução padrão de mãos e paramentação. Através dessa modalidade, os profissionais e estudantes da área da saúde reconhecem Que a infecção hospitalar é favorecida pela falta da adequada lavagem das mãos, o Que, para o Ministério da Saúde, é uma ação Que, isoladamente, constitui-se como a mais importante para se evitar uma infecção cruzada no ambiente hospitalar ${ }^{(13,14,15)}$.

Fator 2 - Lado Inferior (negativo)

No fator 2 (eixo vertical - fonte em negrito) no lado inferior negativo do gráfico, destacam-se as representações sobre infecção hospitalar elaboradas por profissionais e estudantes da área da saúde do sexo masculino, profissionais de nível superior na faixa etária de mais de 50 anos.

As modalidades correspondentes às variáveis de opinião evocadas por esses profissionais e estudantes da área da saúde em resposta ao estímulo infecção hospitalar, esterilização de materiais e precaução padrão, são representadas pelas palavras: limpo, contaminação e paramentação. A limpeza é um procedimento de remoção de sujidade e detritos para manter em estado de asseio os artigos, reduzindo a população microbiana. Já a contaminação consiste na presença de microorganismos sobre a superfície epitelial sem invasão tecidual, reações fisiológicas e dependência metabólica com o hospedeiro ${ }^{(1)}$.

A paramentação é definida como a utilização de barreiras de proteção para pacientes e profissionais à exposição de microorganismos presentes e liberados pelos trabalhadores. Desse modo, configuram-se evocações importantes, visto Que, para se evitar uma infecção cruzada no ambiente hospitalar os profissionais e estudantes da área da saúde, devem adotar medidas para evitar as infecções cruzadas, e a adoção de barreiras de proteção prevenindo a exposição ao sangue e outros fluidos orgânicos, como forma de evitar infecção hospitalar ${ }^{(16-18)}$.

\section{Fator 2 - Lado Superior (positivo)}

No fator 2 (eixo vertical - fonte em negrito) no lado superior positivo do gráfico 1, encontram-se as objetivações de infecção hospitalar, construídas por estudantes da área da saúde, na faixa etária de 20 a 30 anos de idade.

Em resposta aos estímulos, infecção hospitalar, procedimentos invasivos e esterilização de materiais e precaução padrão os sujeitos contribuíram com as modalidades: máscara, luvas, sondagens e mãos, sendo Que sondagem e mãos estão presentes nos dois vetores. A partir dessas evocações, os estudantes evidenciam Que os elementos Que contribuem para infecção hospitalar estão relacionados com o uso adequado dos equipamentos de proteção individual (EPI) e que especialmente para o desenvolvimento dos procedimentos invasivos, a lavagem das mãos deve ser adotada como uma medida importante para a prevenção e controle das infecções hospitalares ${ }^{(15,16,19-21)}$.

\section{CONSIDERAÇÕES FINAIS}

A partir da apreensão das Representações Sociais da Infecção Hospitalar construídas por profissionais e estudantes da área da saúde procurou-se explorar os aspectos envolvidos nesse processo enQuanto determinantes das infecções cruzadas no ambiente hospitalar.

O posicionamento dos sujeitos da pesquisa frente à infecção hospitalar é evidenciado com evocações, tais como: paramentação, sondagem e mãos. Isso reforça Que a ênfase dada pelos profissionais e estudantes da área da saúde à infecção hospitalar está no reconhecimento da importância da paramentação dos Que desenvolvem os procedimentos no ambiente hospitalar para proteger, tanto os pacientes como os profissionais e estudantes de uma infecção, especialmente durante os procedimentos invasivos, ressaltando a importância do cuidado com as mãos. 
Desse modo, percebe-se um posicionamento, visto Que, à medida Que são objetivadas as representações sociais da infecção hospitalar, ancoradas nos aspectos ideológicos, históricos, sociais e sócio-culturais, evidencia-se um conflito entre as propostas do Programa de Prevenção e Controle das Infecções Hospitalares do Ministério da Saúde e o conhecimento Que é socialmente compartilhado entre os profissionais e estudantes da área da saúde sobre a infecção hospitalar, por esse programa focalizar, predominantemente, os aspectos epidemiológicos, biológicos, econômicos, e Quase nunca o ponto de vista social e psicológico.

Portanto, constata-se Que tal programa não contempla a complexidade da comunicação humana, dificultando o alcance dos objetivos propostos e transformando-se em atividades rotineiras e estereotipadas $^{(8)}$. Nesse sentido, não se pode falar em políticas de controle de infecção hospitalar, sem considerar toda uma gama de determinantes das práticas de prevenção e controle das infecções hospitalares, Que se encontram atreladas às crenças, valores, normas sociais, dentre outros.

\section{REFERÊNCIAS}

1. Starling CEF. Vigilância epidemiológica das infecções hospitalares na prática diária. Belo Horizonte (MG): Ed. Cuatiara; 1993.

2. Ministério da Saúde (BR). Portaria no 2616 de 12 de Maio de 1998. Estabelecem diretrizes e normas para a prevenção e controle das infecções hospitalares. Brasília (DF): Ministério da Saúde; 2004.

3. Wagner W. Sócio-gênese e características das representações sociais. In: Moreira ASPM, Oliveira DC. Estudos interdisciplinares de representações sociais. Goiânia (GO): AB; 2000.

4. Abric JC. Méthodologie de recueil des représentations socials. Paris (FR): PUF; 1994.

5. Moscovici S. A representação social da psicanálise. Rio de Janeiro (RI): Zahar; 1978.

6. Jodelet $\mathrm{D}$. As representações sociais: um domínio em expansão. In: Jodelet D. As representações sociais. Rio de Janeiro (RJ): Ed UERJ; 2001 .

7. Jodelet D. Representation sociale: phénomenes concept et théorie. In: Moscovici S. Psychologia sociale. Paris (FRA): Presses Universitaires de France; 1984.

8. Tura LFR, Madeira MC, Gaze R. Representações sociais das hepatites virais e suas implicações nas práticas educativas de prevenção. Cad Saúde Coletiva 2002; 10(2).

9. Coutinho MPL. Representações sociais: abordagem interdisciplinar. João Pessoa (PB): Universitária UFPB; 2003
10. Fernandes A T. Infecção hospitalar e suas interfaces na área da saúde. São Paulo (SP): Atheneu; 2000.

1 I. Lima ML. Fatores sociais nas percepções de riscos. Psicologia 1998; 17(1): 11-28.

12. Ministério do Trabalho (BR). Portaria no 3.214 de 08 de junho de 1978. NR-6. Equipamento de Proteção Individual - EPI. In: Segurança e Medicina do Trabalho. $29^{a}$ ed. São Paulo (SP): Atlas; 1995.

13. Torres S, Lisboa TC. Limpeza e higiene hospitalar. São Paulo (SP): CLR Balieiro; 2001.

14. Yoshitake M. Teoria do controle gerencial. São Paulo (SP): IBRADEM; 2003.

15. Carvalho PR. Boas práticas Químicas em biossegurança. Rio de Janeiro (RJ): Interciência; 1999.

16. Silva MAA. Enfermagem na unidade de centro cirúrgico. São Paulo (SP): EPU; 1997.

17. Bolick D. Segurança e controle de infecção. Rio de Janeiro (RI): Reichmann \& Affonso Editores; 2000.

18. Mastroeni MF. Biossegurança aplicada a laboratórios e serviços de saúde. São Paulo (SP): Atheneu; 2004.

19. Costa MAF, Costa MFB. Biossegurança de A a Z. Rio de Janeiro (RJ): Papel Virtual; 2003.

20. Hirata MH, Filho JM. Manual de Biossegurança. São Paulo (SP): Manole; 2002. 